\title{
THE NATURE OF THE RADIO SOURCE CYGNUS $X$
}

\author{
F. D. DRAKE \\ National Radio Astronomy Observatory \\ Green Bank, West Virginia, U.S.A.
}

The radio source Cygnus $\mathrm{X}$ (IAU 20N4A) has long created interest because of its unusual spectrum, its seemingly extended nature, and its location in a region where we believe .we look nearly lengthwise down a spiral arm of the Galaxy. Piddington and Minnett [1] first showed that the source had a thermal spectrum. In a recent paper Davies [2] has concluded that the source is an extremely large extended $\mathrm{H}$ II region located in the second spiral arm in Cygnus. The recent construction of large parabolic antennas has made it possible to clarify greatly the nature of Cygnus $X$; it is the purpose of this paper to give the results of a high-resolution survey of the Cygnus $\mathrm{X}$ region, and to report a possibly relevant optical observation.

Observations of continuum radiation from a solid angle of about 75 square degrees have been made with the 60 -foot radio telescope at Harvard College Observatory. The antenna beam was nearly circular, with a beamwidth of 49 minutes of arc at half-power points. The receiver was the radiometer built by the Ewen Knight Corporation for the Harvard 24-foot radio telescope [3]. As used here, the radiometer accepted a $2 \mathrm{Mc} / \mathrm{s}$ band of information at a center frequency of $1424.0 \mathrm{Mc} / \mathrm{s}$, and, with only very slight image rejection, an image band of $2 \mathrm{Mc} / \mathrm{s}$ centered on $1366.0 \mathrm{Mc} / \mathrm{s}$. The weighted mean of these frequencies is $1396 \mathrm{Mc} / \mathrm{s}$, in which the weight is given by the relative receiver sensitivity at each frequency; this is considered the effective frequency for the observations reported here. The r.m.s. receiver output fluctuation was about $1^{\circ} \mathrm{K}$ throughout this program. Observations consisted of both right-ascension and declination drift curves across the source at the sidereal rate. The effective integration time and interval at which the curves were read was one minute. Intensity calibrations were obtained by pointing the antenna into a forested hillside, as is done by the Leiden radio group at Kootwijk. At the beginning of this study, calibrations obtained in this manner were compared with those given by a standard gas-tube noise source. These two methods of calibration agreed to within 1 per cent; this apparently high accuracy is probably fortuitous. The antenna gain and side-lobe pattern were determined by a system of observations of the sun and radiation from the earth described by the author [4].

When in the reduction of drift-curve data there is doubt about where the zero-intensity level lies, it is quite advantageous to have declination drift curves besides the usual right-ascension curves. Right-ascension curves have 
no points in common to use in testing for inconsistencies in assigned zero intensity levels. However, if declination drift curves are available, they may be used to " tie together" the right-ascension curves. This, in effect, provides points in common which immediately reveal any inconsistencies in assigned zero-intensity levels.

The observations made provided about 2000 values of antenna temperature. Arbitrarily assigned zero levels were then adjusted slightly by trial and error until the right-ascension and declination drift-curve data were all mutually consistent, and a radio contour map was drawn. These observational results are shown in Fig. 1. Recently, Westerhout [5] published a similar radio map of the Cygnus $X$ region, made at a frequency of $1390 \mathrm{Mc} / \mathrm{s}$ with the 25 -meter telescope at Dwingeloo. His map and the one shown here, both of which are completely independent studies, are in extremely good agreement. What minor differences exist between them may be attributed to the difference between the two antennas used. It is quite clear from these results that Cygnus $\mathrm{X}$ is not a single large extended source, but rather a complex of many discrete sources.

Before making further analysis of this data, it is necessary to correct for antenna effects. These are quite serious in the present case, in which many

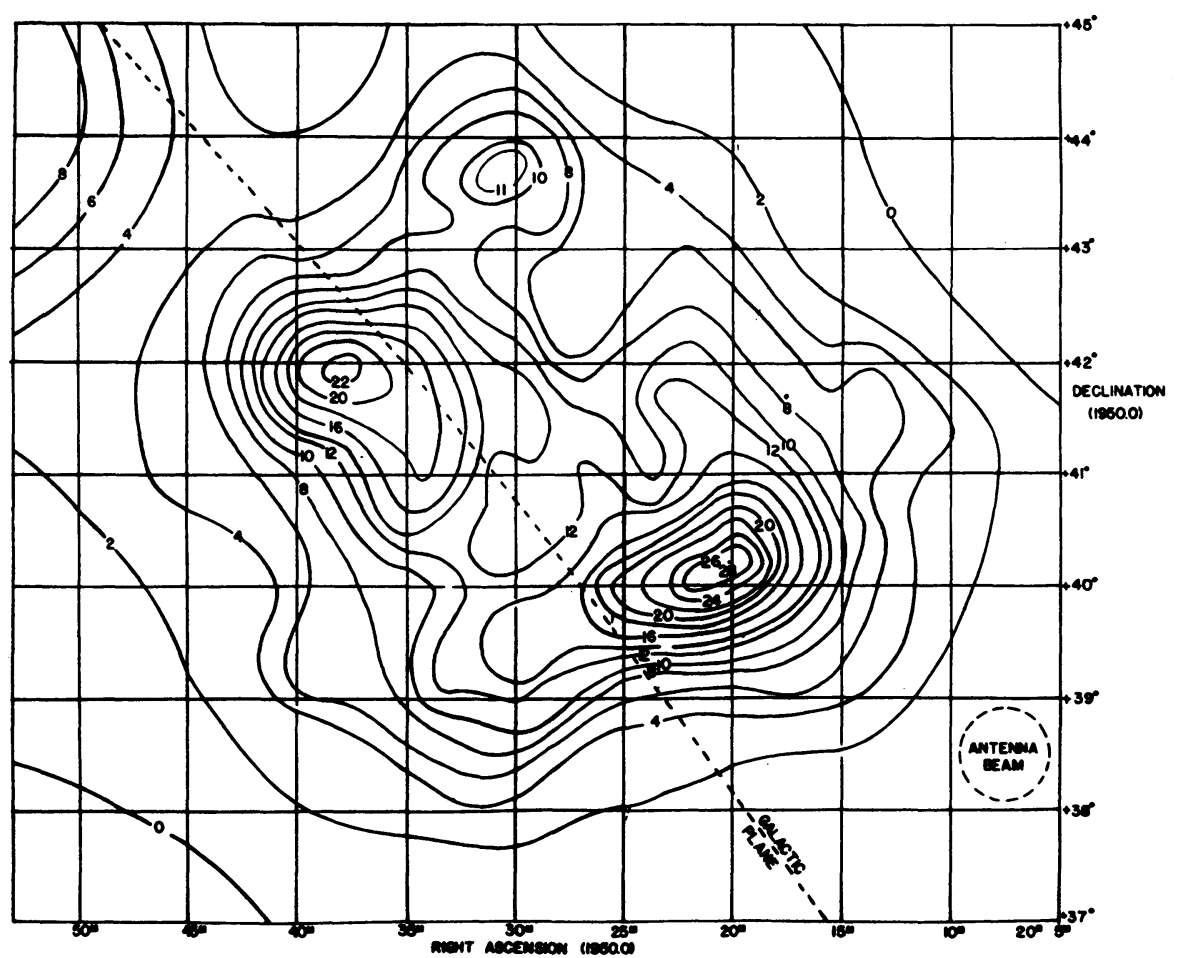

Fig. 1. Region of Cygnus X. Plot of antenna temperatures in ${ }^{\circ} \mathrm{K}$, Harvard 60 -foot radio telescope, $\lambda 21.5 \mathrm{~cm}$. 
sources are in close mutual proximity. Accordingly, the antenna temperature contours have been corrected for side-lobe effects and antenna gain, using a technique given by the author [4], and then corrected for antenna main-beam smoothing, using the technique developed by Bracewell [6]. The result is a map of radio-brightness temperature shown in Fig. 2, which should contain the maximum information obtainable with a 60 -foot antenna and the observing time and receiver sensitivity used here. It has often been speculated that Cygnus $\mathrm{X}$ is an $\mathrm{H}$ il region connected with the association VI Cygni, whose position has been plotted in Fig. 2. As is seen, the association, which is near the center of the Cygnus $\mathrm{X}$ complex, lies very near the position of minimum radiation from the complex. It is evident from this that VI Cygni can be responsible for at most only a small fraction of the Cygnus $\mathrm{X}$ radio emission.

The problem of source identification is almost entirely solved by comparing Fig. 2 with a high-quality $\mathrm{H} \alpha$ photograph of the region. This is shown in Fig. 3, in which the $\mathrm{H}$ II regions visible in the Palomar Sky Atlas have been plotted on a simplified version of Fig. 2. It is seen that, in nearly every case, an $\mathrm{H}$ II region is associated with the observed radio source. In some cases the radio-brightness contours follow almost exactly the outlines of visible

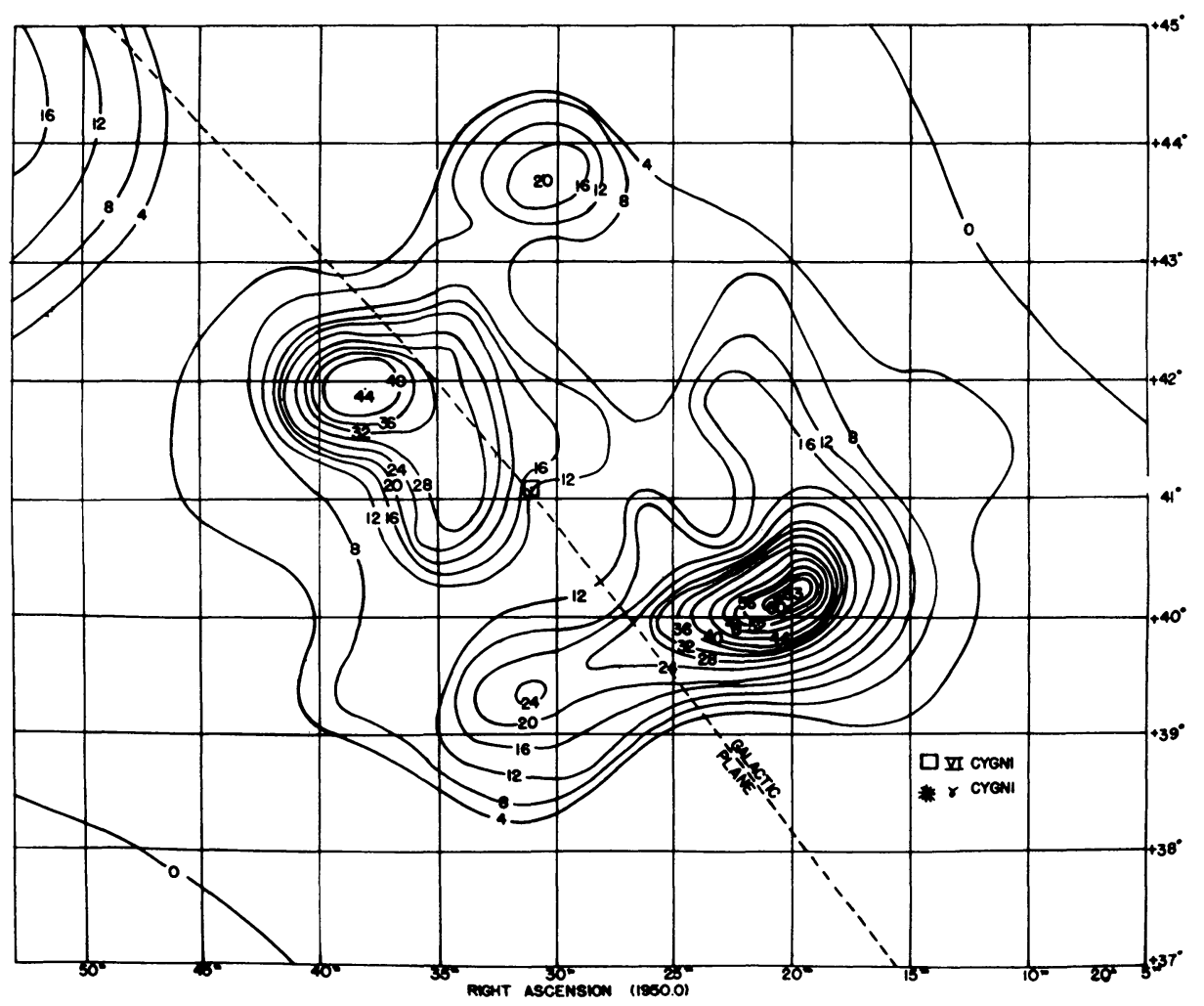

FIG. 2. Region of Cygnus X. Radio brightness temperatures in ${ }^{\circ} \mathrm{K}, \lambda 21.5 \mathrm{~cm}$. 


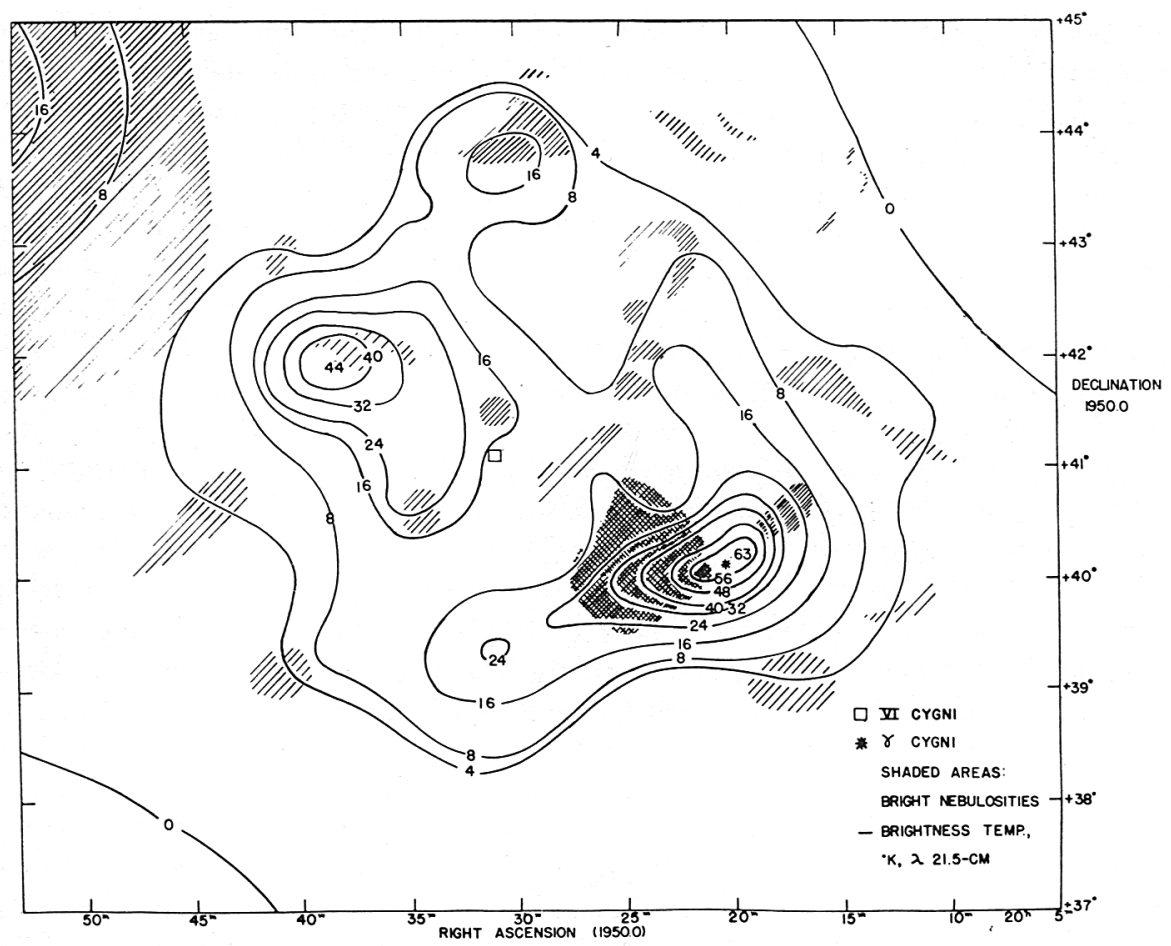

FIG. 3. Region of Cygnus X. Radio brightness contours, $\lambda 21.5 \mathrm{~cm}$, and bright nebulosities.

$\mathrm{H}$ II regions. It is quite evident that most of the Cygnus $\mathrm{X}$ emission arises from an unusual number of separate $\mathrm{H}$ in regions concentrated near the same apparent position. Other than the strongest source, only one strong source, at right ascension $20^{\mathrm{h}} 31^{\mathrm{m}} \cdot 1$, declination $+39^{\circ} 20^{\prime}$, has no visible nebulosity connected with it, which is to be expected, as this source lies in the most obscured portion of the Cygnus Rift. The catalog designations of the nebulae associated with Cygnus $\mathrm{X}$ have been tabulated by Westerhout [5].

The data of Fig. 2 may be used to compute some of the physical parameters for the nebulae concerned. First, however, it is necessary to make some estimate of the distances to the nebulosities. In no case can the nebularexciting star or stars be found with certainty in the existing catalogs, the most reliable source of distances thus being eliminated. An alternate source of distance values is $21-\mathrm{cm}$ absorption measurements of the sources concerned. Davies [2] has presented observational evidence leading him to conclude that there is a deficiency of $\mathrm{HI}$ in the second spiral arm in Cygnus, and that absorption of Cygnus $X$ radiation occurs in the first spiral arm. This absorption is indicated by a decrease in the intensity of the $21-\mathrm{cm}$ profile component associated with the first spiral arm, and would place the stronger Cygnus $X$ components beyond that arm. In the course of studying the Cygnus region, Muller and Westerhout [7] and Mrs. Kassim [8] have taken profiles in nearly 
the same positions as Davies' profiles. These do not support Davies' results. The Leiden and Harvard results were both made with antennas nearly equal in size to Davies' antenna, and are in extremely good mutual agreement on the points significant here: they show no $\mathrm{H}_{\mathrm{I}}$ deficiency in the second spiral arm, and no absorption effect in the first spiral arm, but rather an increase in the profile amplitude in the position of Cygnus $\mathrm{X}$. Considering these results, it does not seem appropriate to use existing $21 \cdot \mathrm{cm}$ data to determine distances to the Cygnus $\mathrm{X}$ nebulosities. The only available datum bearing on the nebular distances, then, is the fact that all the visible nebulosities associated with strong radio emission seem to be obscured by the Cygnus Rift. The distance to the Rift is about 1250 parsecs [9] ; therefore, the minimum distance to the nebulosities is of this order. We will assume in our calculations that the minimum distance, $r$, is 1500 parsecs, which will allow easy comparison with Westerhout's results.

From Fig. 2, it is possible to determine directly an apparent source diameter $\theta_{r}$ and mean brightness temperature $T_{b}$ for each source. In determining the mean brightness temperature, the average temperature of the background in the source vicinity must be subtracted from the brightness temperature at the source position. The accuracies to which these two parameters may be determined depend on the strength of a source, and the degree to which it is blended with other sources; it appears improbable that the error in either parameter is greater than a factor of two with any source, and in most cases the errors are probably considerably less. From these two parameters, one may compute the mean emission measure $E$, the total source flux $S$, the electron density $n$, and the total ionized mass $M$. We assume a model for the nebula consisting of a uniform cylinder of matter of diameter and length $\theta_{r} r$. Because we have only a lower limit on $r$, we will obtain only an upper limit on $n$ and lower limit on $M$. For a frequency of $1396 \mathrm{Mc} / \mathrm{s}$, formulas giving the desired values are [5];

$$
\begin{gathered}
E=546 T_{b} ; \\
n=\frac{48}{\theta_{r}} \sqrt{\frac{S\left(10^{26}\right)}{r \theta_{r}}} \quad \text { electrons } / \mathrm{cm}^{3} ; \\
M=4.8\left(10^{-6}\right) \theta_{r} r^{2}\left(\theta_{r} r S 10^{26}\right)^{1 / 2} \odot
\end{gathered}
$$

Here, $T_{b}$ is expressed in ${ }^{\circ} \mathrm{K}, \theta_{r}$ in degrees, $r$ in parsecs, $S$ in watts $/ \mathrm{m}^{-2}(\mathrm{c} / \mathrm{s})^{-1}$, and it has been assumed that the electron temperature is $10^{4} \mathrm{~K}$. The results of this analysis of Fig. 2 are given in Table I.

The results of Table I are in good agreement with the similar results given by Westerhout. Most of the differences that occur between the two sets of results may be traced eventually to the difference in size between the two antennas, which causes different degrees of source-blending to occur in each survey. Correction for this blending, which cannot be exact, leads to slightly different source diameters and brightnesses in each survey, which in turn lead to differences in the derived nebulosity parameters. In general, the 
differences between corresponding pairs of values from the two sets of results are of one sign about as much as the other sign; there is no marked systematic difference between the two sets of results. Probably the most significant general result given by Table I is the fact that we deal mostly with extremely massive nebulosities. Larger telescopes and more sensitive receivers will be required to detect the numerous small nebulosities known to exist.

TABLE I

\begin{tabular}{|c|c|c|c|c|c|c|c|c|c|}
\hline Source & $\begin{array}{l}\text { Westerhout } \\
\text { Number }\end{array}$ & $\begin{array}{c}\alpha \\
(1950.0)\end{array}$ & $\stackrel{\delta}{\delta}$ & $\theta_{r}$ & $\begin{array}{l}\boldsymbol{T}_{b} \\
{ }^{\circ} \mathbf{K}\end{array}$ & $E$ & ${ }_{10-26} \stackrel{S}{\text { M.K.S. }}$ & $\begin{array}{l}n_{\max } \\
\mathrm{cm}^{-3}\end{array}$ & $\underset{0}{M_{\min }}$ \\
\hline 1 & 62 & $20^{\mathrm{h}} 13 \mathrm{~m} 0$ & $+41^{\circ} 30^{\prime}$ & $1^{\circ}$ & 4 & 2200 & 60 & 10 & 3200 \\
\hline 2 & 66 & 2019.7 & 4017 & .75 & 50 & 27000 & 400 & $*$ & $t$ \\
\hline 3 & - & $\begin{array}{ll}20 & 21.7\end{array}$ & 4208 & .7 & 10 & 5500 & 70 & 18 & 2000 \\
\hline 4 & 67 & 2025.8 & 3954 & .75 & 20 & 11000 & 60 & 24 & 3400 \\
\hline 5 & 68 & 2025.8 & 4044 & .5 & 8 & 4500 & 30 & 19 & 800 \\
\hline 6 & - & 2030.3 & 4130 & .6 & 6 & 3300 & 30 & 15 & 1100 \\
\hline 7 & 70 & 2030.4 & 4344 & 1.2 & 12 & 6500 & 250 & 15 & 8700 \\
\hline 8 & 69 & 2031.1 & 3920 & 1.3 & 14 & 7600 & 240 & 13 & 9500 \\
\hline 9 & 72 & 2034.0 & 4244 & .6 & 8 & 4000 & 40 & 19 & 1200 \\
\hline 10 & 73 & 2034.2 & 4102 & 1.0 & 16 & 8700 & 230 & 19 & 6300 \\
\hline 11 & 75 & 2038.1 & 4155 & 1.1 & 36 & 20000 & 640 & 27 & 12000 \\
\hline 12 & 76 & 2039.4 & 3920 & .8 & 6 & 3000 & 50 & 12 & 2100 \\
\hline
\end{tabular}

It remains to discuss the strongest component of Cygnus $\mathrm{X}$, which is not as simply interpreted as the other components. This source lies very close to the star $\gamma$ Cygni, in a heavily obscured region where no bright nebulosity is visible. Furthermore, the source is strongly blended with several nearby sources, which prevents an accurate determination of the source position or size. A photograph of the region is shown in Fig. 4, on which the source positions found in this and Westerhout's survey have been marked. Fig. 4 shows the existence of an unusual structure of dark nebulosities surrounding $\gamma$ Cygni. They appear as a circular dark-ring structure, nearly concentric with the stellar image, and are best seen southeast of the star, where the nebulosities lie just outside the edge of the stellar image. This structure may be followed from about position angle 0 to $270^{\circ}$ with respect to $r$ Cygni. It is not clear which, if any, nebulosities in the remaining quadrant belong to this system. Visible also is a system of faint-emission nebulosities extending from the stellar image, and enclosed in the dark ring, which give the stellar image a somewhat "spoked" appearance. Westerhout's position for the strongest Cygnus $\mathrm{X}$ component, which is probably more accurate because it was made with the narrower beamwidth, falls within this system of nebulosities. The position found in this study probably falls within the nebulosities; the positions point to the existence of a source very near $r$ Cygni.

An $\mathrm{H} \alpha$ photograph has been made of $\gamma$ Cygni with the Harvard 61-inch reflector; it is shown in Fig. 5. This shows clearly the existence of a very 


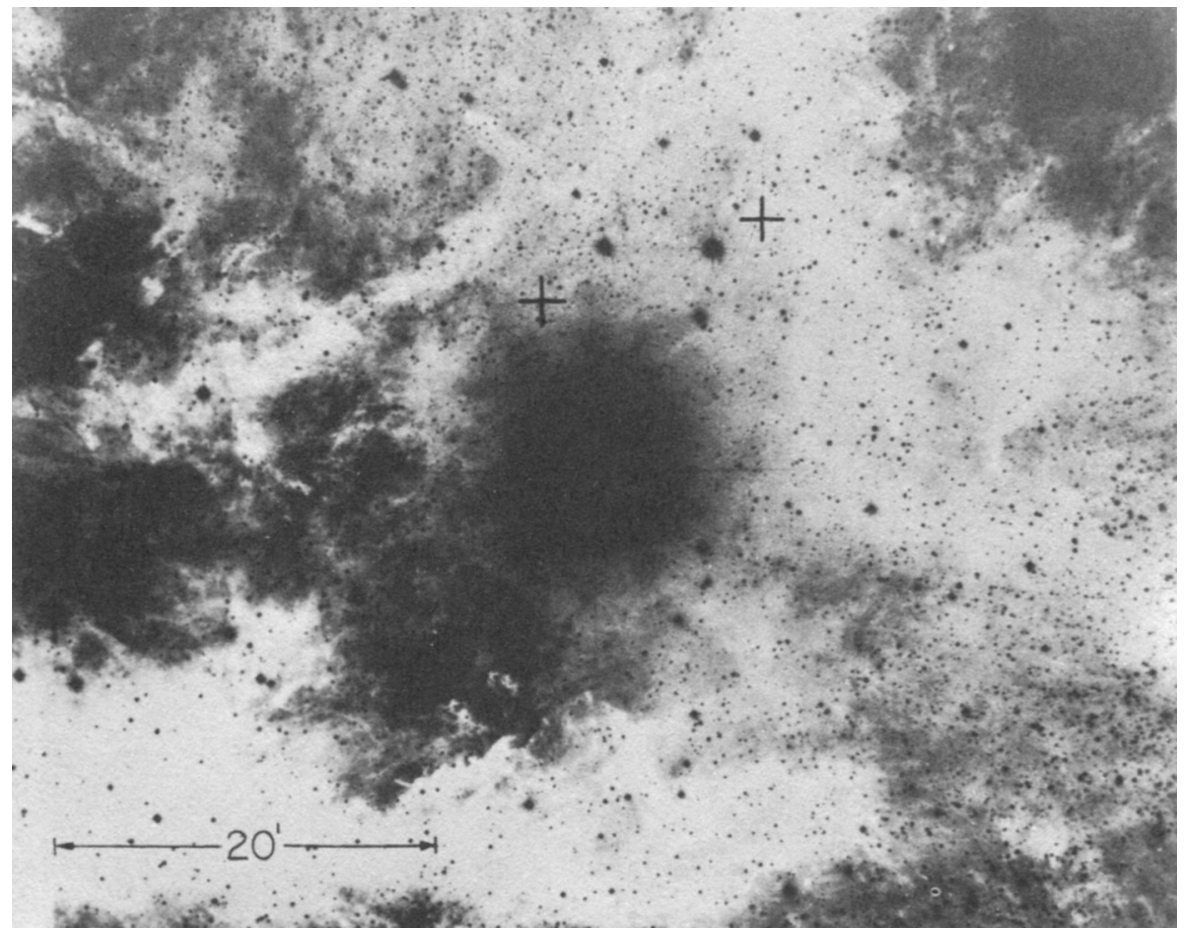

Fig. 4. Palomar Sky Atlas red plate of region of $\gamma$ Cygni. The left cross is the position of the brightest Cygnus $\mathrm{X}$ component as found by Westerhout, the right cross is the position found in this study. East is to the left, north to the top.

bright nebula so near the star that it was obscured by the stellar image in Fig. 4. Comparison of this nebula with others visible on both Fig. 4 and 5 suggests that it is as bright as or brighter than any other nebulae in the region. A $103 a-O$ plate of the same region made with the same telescope and giving the same density in the $r$ Cygni image shows no trace of the nebula. This makes it appear very probable that the nebulosity is, indeed, an $\mathrm{H}$ II region and not a reflection nebula, although Minkowski [10] has suggested that one must still explore the possibility that this is a heavily reddened reflection nebula. The proximity of the measured radio-source position to the nebula suggests that the nebula is the radio source. If this is the case, with $r$ Cygni at a distance of 100 parsecs, $n$ is of the order of $150 \mathrm{atoms} / \mathrm{cm}^{3} . M$ is given as 60 by Eq. (3). This is undoubtedly a high upper limit, as the nebula clearly appears to consist of many bright knots. These are reasonable values; it is surprising, however, that an F8 $\mathrm{I} b$ star could excite such a nebulosity. Higher-resolution measurements of the position and size of the radio source must be made before it may be decided whether this nebula is the radio source, or only an interesting nebulosity that has been brought to attention by a chance coincidence with a bright radio source. 


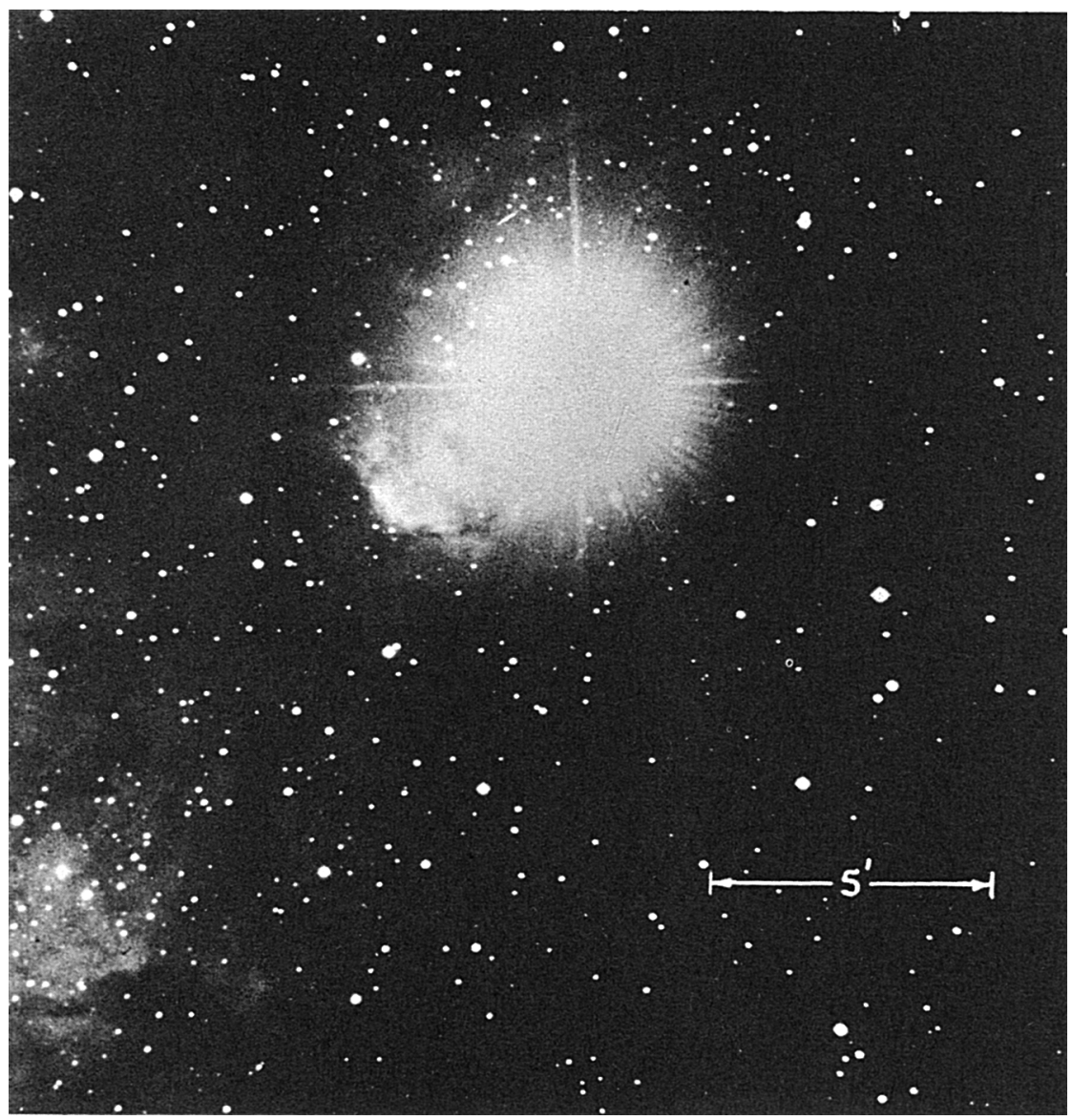

Frg. 5. Region of $\gamma$ Cygni in H $\alpha$ and [N II] light. Harvard 61-inch reflector, 103 $=$ E plate with Corning 2403 filter. Exposure $3^{\mathrm{h}} 30^{\mathrm{m}}$, 1956 November 4 . East is to the left, north to the top.

\section{REFERENCES}

[1] Piddington, J. H., and Minnett, H. C. Aust. J. Sci. Res. A 5, 17, 1952.

[2] Davies, R. D. M.N.R.A.S. 117, 663, 1957.

[3] Lilley, A. E. Ap. J. 121, 559, 1955.

[4] Drake, F. D. Thesis, Harvard University, 1958.

[5] Westerhout, G. B.A.N. 14, 215, 1958.

[6] Bracewell, R. N. Aust. J. Phys. 8, 54, 1955.

[7] Muller, C. A., and Westerhout, G. B.A.N. 13, 151, 1957.

[8] Kassim, M. A. K. Thesis, Radcliffe College, 1958.

[9] Miller, F. D. Harvard Annals, 105, 297, 1937.

[10] Minkowski, R. Private communication, 1957. 